\title{
More than just perception-action recalibration: Walking through a virtual environment causes rescaling of perceived space
}

\author{
Jonathan W. Kelly • Lisa S. Donaldson • \\ Lori A. Sjolund • Jacob B. Freiberg
}

Published online: 10 July 2013

(C) Psychonomic Society, Inc. 2013

\begin{abstract}
Egocentric distances in virtual environments are commonly underperceived by up to $50 \%$ of the intended distance. However, a brief period of interaction in which participants walk through the virtual environment while receiving visual feedback can dramatically improve distance judgments. Two experiments were designed to explore whether the increase in postinteraction distance judgments is due to perception-action recalibration or the rescaling of perceived space. Perception-action recalibration as a result of walking interaction should only affect action-specific distance judgments, whereas rescaling of perceived space should affect all distance judgments based on the rescaled percept. Participants made blind-walking distance judgments and verbal size judgments in response to objects in a virtual environment before and after interacting with the environment through either walking (Experiment 1) or reaching (Experiment 2). Size judgments were used to infer perceived distance under the assumption of size-distance invariance, and these served as an implicit measure of perceived distance. Preinteraction walking and size-based distance judgments indicated an underperception of egocentric distance, whereas postinteraction walking and size-based distance judgments both increased as a result of the walking interaction, indicating that walking through the virtual environment with continuous visual feedback caused rescaling of the perceived space. However, interaction with the virtual environment through reaching had no effect on either type of distance judgment, indicating that physical translation through the virtual environment may be necessary for a rescaling of
\end{abstract}

J. W. Kelly • L. S. Donaldson • L. A. Sjolund · J. B. Freiberg

Iowa State University, Ames, IA, USA

J. W. Kelly $(\square)$

Department of Psychology, Iowa State University, W112

Lagomarcino Hall, Ames, IA 50011-3180, USA

e-mail: jonkelly@iastate.edu perceived space. Furthermore, the size-based distance and walking distance judgments were highly correlated, even across changes in perceived distance, providing support for the sizedistance invariance hypothesis.

Keywords Visual perception · Distance perception . Virtual reality $\cdot$ Action

Immersive virtual reality technology has numerous applications, including physical and psychological rehabilitation, education, training, entertainment, and human behavioral research. In order to be fully effective in these applications, virtual environments should be perceived as realistic representations of actual environments. One consistent challenge in creating realistic virtual environments is the tendency for underperception of distances, resulting in virtual environments that appear smaller than their real-world counterparts.

In real environments under full-cue viewing, the perception of egocentric distance - the distance from oneself to an object - can be quite accurate. Perceived egocentric distance has been measured in multiple ways, including verbal numerical report (da Silva, 1985; Foley, 1977; Gibson \& Bergman, 1954), "blind" walking (Loomis, da Silva, Fujita, \& Fukusima, 1992; Loomis, Klatzky, Philbeck, \& Golledge, 1998; Rieser, Ashmead, Talor, \& Youngquist, 1990; Sinai, Ooi, \& He, 1998) and throwing (Eby \& Loomis, 1987; Ooi, $\mathrm{Wu}, \& \mathrm{He}, 2001)$ to a previously viewed location, and size judgment, which is used to infer perceived distance (Gogel, Loomis, Newman, \& Sharkey, 1985; Hutchison \& Loomis, 2006; Sedgwick, 1986). Action-based measures such as walking and throwing typically produce more accurate responses than do verbal measures, which show a tendency toward underestimation of distance (see Loomis \& Philbeck, 2008, for a review). The slopes relating judged distance to actual 
distance average around 0.8 for verbal responses and 1.0 for action-based responses (Loomis \& Philbeck, 2008). It is possible that the different response types could be based on unique internal representations. However, scaling verbal distance judgments by a constant creates a tight correspondence between locations judged through verbal report and blind walking (Loomis \& Philbeck, 2008), and both verbal and blind-walking judgments are similarly influenced by manipulations of egocentric distance cues (Philbeck \& Loomis, 1997), suggesting that both judgment types might operate on the same percept.

Action-based judgments of egocentric distances in the physical world have been found to be nearly $100 \%$ of the actual distance for distances up to $20 \mathrm{~m}$ (for a review, see Loomis \& Knapp, 2003). In comparison, action-based judgments of egocentric distance in virtual environments are commonly only $50 \%-85 \%$ of the modeled distance (i.e., the distance intended by the designer of the virtual environment; Bodenheimer et al., 2007; Gooch \& Willemsen, 2002; Kelly, Beall, \& Loomis, 2004; Knapp \& Loomis, 2004; Kuhl, Thompson, \& Creem-Regehr, 2009; Messing \& Durgin, 2005; Steinicke et al., 2009; Thompson et al., 2004; Waller \& Richardson, 2008; Willemsen, Colton, Creem-Regehr, \& Thompson, 2009; Willemsen, Gooch, Thompson, \& CreemRegehr, 2008; Witmer \& Sadowski, 1998; Ziemer, Plumert, Cremer, \& Kearney, 2009).

The cause of distance underperception in virtual environments is not well understood. One approach to resolving the problem has been to identify which distance cues are missing from the virtual environment or the display technology, in hopes of improving the deficient cues. This approach is based on the fact that the removal of certain cues from real-world viewing, such as when displaying luminous objects in an otherwise dark room, causes overperception of near distances and underperception of far distances, resulting in an overall compression of the range of response distances relative to the range of the stimulus distances (Philbeck, Loomis, \& Beall, 1997; Tresilian, Mon-Williams, \& Kelly, 1999). Therefore, the misperception of distance in virtual environments may be due to one or more missing or unreliable distance cues. To that end, past research on distance perception in virtual environments has examined the contributions of distance cues that are thought to be affected by low-quality computer graphics (Thompson et al., 2004), reduced field of view (Knapp \& Loomis, 2004; Willemsen et al., 2009), inaccurate stereoscopic rendering (Willemsen et al., 2008), and the mass and inertia of head-mounted displays (HMDs; Willemsen et al., 2009). Although progress has been made toward understanding the cues that affect distance perception in virtual environments, none of the aforementioned cues can fully account for the distance underperception in virtual environments.

In contrast to the cue-based approach, another approach to resolving the problem of distance underperception has been to allow viewers to interact with the virtual environment and receive feedback about their actions. Multiple studies have shown that a brief period of interaction, in which participants walk to various locations within the virtual environment while receiving visual feedback, can cause judgments of egocentric distance in the virtual environment to increase by up to $70 \%$ (Mohler, Creem-Regehr, \& Thompson, 2006; Richardson \& Waller, 2005, 2007; Waller \& Richardson, 2008). This increase in postinteraction judgments appears to require physically walking through the virtual environment. Distance judgments were unaffected by interactions in which participants viewed simulated visual walking (i.e., optic flow only; Waller \& Richardson, 2008), indicating the necessity of body-based movement. Furthermore, distance judgments increased after participants performed a blind-walking task in which they received visual or verbal feedback (Mohler et al., 2006; Richardson \& Waller, 2005), indicating the sufficiency of body-based interaction and accompanying offline feedback.

At least three possible mechanisms might allow walking in the virtual environment to cause an increase in postinteraction distance judgments. First, participants may develop an explicit strategy, whereby strategic adjustments are made in order to produce more accurate responses despite underperception of the target distance. One way in which this explicit strategy could be instantiated would be to modify the response output. An example of such an explicit strategy would be, "Walk twice as far as the object appears" (Richardson \& Waller, 2005). This strategy is herein referred to as the response modification strategy. The response modification strategy can readily explain an increase in direct blind-walking judgments, in which participants walk a direct path to the previously viewed object. However, interaction with the virtual environment not only improves direct blind-walking judgments, but also improves indirect blind-walking judgments, in which participants walk along an oblique path before turning and walking to the target (Richardson \& Waller, 2007). Such generalization to other walking responses is unlikely to occur when using a response modification strategy. An alternative formulation of the explicit strategy is the representation modification strategy, whereby the perceptual representation itself is explicitly modified to correct for underperception of egocentric distance. Explicit modification of the percept itself may be unlikely, but modification of an internal representation that persists after vision is occluded (like the "spatial image" described by Loomis, Klatzky, \& Giudice, 2013) seems more plausible. Since the representation modification strategy would result in a new, corrected representation, any response based on the new representation should reflect the same correction that was initially applied, which could explain findings of generalization across response types (e.g., from direct walking responses to indirect walking responses; Richardson \& Waller, 2007). However, it is unlikely that the corrected representation would also lead to more accurate (i.e., increased) size judgments, since most naive participants would 
be unaware of the relationship between perceived size and perceived distance. ${ }^{1}$

A second possible mechanism that could account for increased postinteraction distance judgments is that interaction with the virtual environment may cause perception-action recalibration (Antis, 1995; Bruggeman, Pick, \& Rieser, 2005; Durgin et al., 2005; Mohler et al., 2007; Rieser, Pick, Ashmead, \& Garing, 1995). The recalibration hypothesis is that walking through the virtual environment with visual feedback modifies the perception-action coupling (Richardson \& Waller, 2007; Waller \& Richardson, 2008), such that recalibration changes the imagined rate of movement through the environment during subsequent blind walking. Recalibration of walking has been shown to transfer to other translational movements, such as crawling (Withagen \& Michaels, 2002) and side-stepping (Rieser et al., 1995), but it does not transfer to different categories of responses, such as throwing (Rieser et al., 1995). Taken together, these findings support the functional account of recalibration (Rieser, 1999; Rieser et al., 1995), whereby recalibration transfers within a functional category of action, such as translation or rotation, but not across categories. This functional account of recalibration also fits well with the finding that recalibration of forward walking changes blind-walking distance judgments but does not change biomechanical aspects of walking, such as step length (Rieser et al., 1995).

A third possible mechanism to account for increased postinteraction distance judgments is that interaction with the virtual environment may cause rescaling of perceived space, whereby the virtual environment appears larger after interaction. As was suggested by Waller and Richardson (2008), rescaling of perceived space might occur if interaction with the virtual environment results in perceptual learning, such that participants learn to attend to distance cues that had previously been ignored or weighted inappropriately. If interaction changes the perceived scale of the virtual environment, any type of distance judgment should be affected, including indirect walking, throwing, and verbal report of distance or size. A rescaling of perceived space may be indistinguishable from global recalibration of all potential actions, but we prefer the rescaling concept for ease of exposition when comparing it with recalibration of a single action, and because it offers a more parsimonious explanation.

The present project was designed to distinguish between the representation modification strategy, the perception-action recalibration hypothesis, and the rescaling hypothesis, all of which could account for the results of existing studies showing that walking through a virtual environment with visual feedback causes an increase in postinteraction blind-

\footnotetext{
${ }^{1}$ Most people, when asked about the relationship between perceived size and perceived distance, report that objects that are farther away look smaller, and therefore that underperception of distance should cause objects to appear larger (in fact, the opposite is true).
}

walking distance judgments (Richardson \& Waller, 2005, 2007; Waller \& Richardson, 2008). In two experiments, participants made direct blind-walking judgments and size judgments before and after interacting with the virtual environment. Size judgments are considered to be a more indirect measure of perceived distance than are blind-walking distance judgments or verbal distance judgments. According to the size-distance invariance hypothesis (Gilinsky, 1951; Gogel et al., 1985; Hutchison \& Loomis, 2006; Sedgwick, $1986)$, perceived object size $\left(S^{\prime}\right)$ is directly related to perceived object distance $\left(D^{\prime}\right)$ and angular size $(\alpha)$ :

$S^{\prime}=2 D^{\prime} \times \tan (\alpha / 2)$.

For objects of equal angular size, those that appear farther away will also appear larger, and vice versa. Furthermore, perceived size can be used to determine perceived distance. Distance estimates inferred through verbal size judgments are herein referred to as size-based distance judgments.

Some researchers have questioned the direct relationship between perceived size and perceived distance, noting, for example, that increasing the distance to an object of constant physical size causes an increase in perceived object size that exceeds what would be expected on the basis of perceived object distance (see Epstein, Park, \& Casey, 1961). However, this failure of size-distance invariance may, in some cases, be due to additional complexities in judgments of perceived distance, especially under reduced-cue viewing. For example, in the absence of any depth cues, the perceived egocentric distance of an object is approximately $2 \mathrm{~m}$ (Gogel, 1969; Gogel \& Tietz, 1973), a finding known as the specific distance tendency. Gogel (1971) suggested that reported failures of size-distance invariance (e.g., Epstein \& Landauer, 1969) could be due to the effect of the specific distance tendency on size and distance judgments. Adding further complexity to the size-distance invariance hypothesis, Oyama (1974) reported that judgments of perceived size and distance correlated highly with the object's visual angle as well as the binocular convergence angle when viewing the object, but that perceived size and perceived distance were not directly related to one another. Despite these theoretical challenges, the use of perceived size as an indirect measure of perceived distance was justified in the present experiments by the need for an implicit measure of perceived distance and the high correlation reported between size-based distance judgments and other direct (Hutchison \& Loomis, 2006) and indirect (Gogel et al., 1985) measures of perceived distance.

In Experiment 1, we examined whether interaction with the virtual environment through walking would cause an increase in size-based distance judgments, or whether the effects of interaction would be limited to walking judgments. In other words, Experiment 1 was designed to investigate whether the effects of walking interaction would transfer to 
an implicit and nonaction type of distance judgment. In Experiment 2, we examined whether interaction with the virtual environment through reaching would cause an increase in walking and/or size-based distance judgments. The previous research has been limited to interaction through walking or simulated walking (optic flow only), and Experiment 2 was designed to extend that work by determining the limits of body-based interactions and their effects on distance judgments in virtual environments.

\section{Experiment 1}

The participants in Experiment 1 made direct blind-walking judgments and size judgments in response to objects viewed in a virtual environment both before and after walking through the environment. With the exception of the size judgments, Experiment 1 was closely modeled on a study described by Waller and Richardson (2008; the "body-based + optic flow" condition of their Exp. 2). If walking interaction causes explicit modification of the representation or recalibration between a visual input and the action output, but does not alter the perceived scale of space, then walking distance judgments, but not size-based distance judgments, should increase after interaction. If walking through a virtual environment causes rescaling of perceived space (i.e., alteration of percepts, such that the virtual environment appears larger after the interaction), then walking distance judgments and size-based distance judgments should both increase after interaction.

\section{Method}

Participants A group of 16 students at Iowa State University participated in exchange for course credit. Two of the participants withdrew from the experiment after experiencing simulator sickness. The data from one participant were removed from all analyses due to size judgments that were not highly correlated with actual object size $(r=.56)$, relative to the average correlation between actual and judged size across all other participants $(M=.96, S E=.007)$.

Stimuli and design Our participants made direct blindwalking judgments and size judgments before interacting (preinteraction) and after interacting (postinteraction) with a virtual environment. When making walking judgments and size judgments, virtual objects were viewed from a fixed viewing position marked on the floor by a rubber strip that could be sensed through the feet. The virtual environment consisted of an infinitely large ground plane covered with a grass texture. A vertical red cylinder (Fig. 1a) on the ground plane served as the stimulus during the pre- and postinteraction walking judgments, and also served to guide participants'
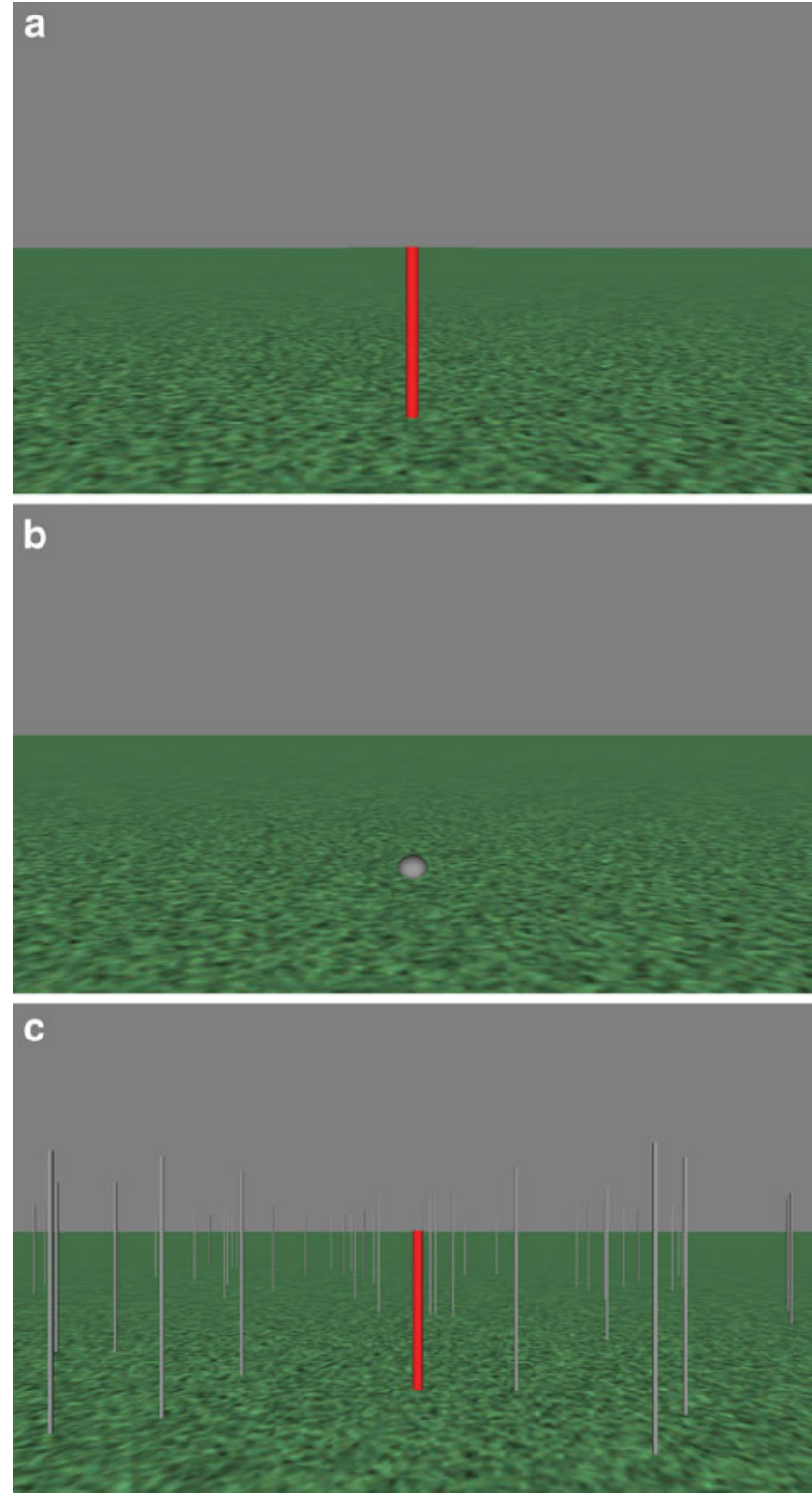

Fig. 1 Perspective views of the virtual environment used in Experiment 1 during the walking judgments (a), the size judgments (b), and the walking interaction (c)

walking movements during the interaction phase. The diameter of the cylinder was $0.1 \mathrm{~m}$, and the height of the cylinder was continuously scaled to match the participant's eye height. A gray sphere (Fig. 1b) placed on the ground plane served as the stimulus during pre- and postinteraction size judgments. During the interaction phase, 150 gray cylinders (Fig. 1c), each $0.03 \mathrm{~m}$ in diameter and $2.5 \mathrm{~m}$ in height, were placed in random locations within a $30 \times 30 \mathrm{~m}$ area in order to enhance optic flow during walking.

During the preinteraction phase, participants made walking judgments and size judgments in response to objects placed at egocentric distances of $0.75,1.25,2.25,3.0,3.5$, and $4.25 \mathrm{~m}$. 
The object diameters used during the preinteraction size judgments were $0.11,0.14,0.2,0.26$, and $0.29 \mathrm{~m}$. During the postinteraction phase, participants made walking judgments and size judgments in response to objects placed at egocentric distances of $0.9,1.45,2.5,3.15,3.9$, and $4.1 \mathrm{~m}$. The object diameters used during postinteraction size judgments were $0.09,0.16,0.2,0.24$, and $0.31 \mathrm{~m}$. During the interaction phase, participants walked with vision to the red cylinder, which appeared at one of the same egocentric distances used in the preinteraction phase.

Participants made 18 walking judgments (three repetitions of six object distances) and 30 size judgments (all possible combinations of six object distances and five object sizes) during both the pre- and postinteraction phases. The walking judgments and size judgments were blocked, and the block order was counterbalanced. Object distance and size were randomized within each block. During the interaction phase, participants walked to 18 objects (three repetitions of the six object distances used in the preinteraction phase).

The virtual environment was viewed on an HMD (nVisor SX111, NVIS, Reston, VA), which provided binocular images presented at a resolution of $1,280 \times 1,024$ pixels within a $102^{\circ}$ (horizontal) $\times 64^{\circ}$ (vertical) field of view. The graphics were updated at $60 \mathrm{~Hz}$ and reflected changes in the participant's head position and orientation. Thus, minor head movements during distance and size judgments could have provided depth information through motion parallax, although no participants were observed to be explicitly using this as a strategy by making unusually large head movements. Graphics were rendered using Vizard software (WorldViz, Santa Barbara, CA) running on a computer with Intel Core2 Quad processors and Nvidia GeForce GTX 285 graphics card.

Procedure After providing informed consent, each participant was given a brief description of the walking judgment and size judgment procedures and was then shown a ruler as a reminder of the standard units of measurement to be used when making size judgments (the standard had both metric and imperial units marked). The participant walked to the viewing position and donned the HMD, and the room lights were extinguished.

For walking judgments, the red cylinder appeared in front of the participant for $5 \mathrm{~s}$, after which the screen went blank, indicating that the participant should walk to the perceived location of the previously seen cylinder. The experimenter pressed a key to log the participant's head position after completion of the walking response. The participant was then led back to the viewing position, and the next trial ensued.

For size judgments, the gray sphere appeared in front of the participant and remained visible until the participant verbally reported the object's diameter. Participants were instructed to report sizes using the units with which they were most familiar. Most responses were given in inches and were later converted to meters prior to the analysis. After the response was recorded, the screen went blank for $1 \mathrm{~s}$ before the next sphere appeared.

During the interaction phase, the participant walked to the location of the red cylinder. The virtual environment remained visible throughout the interaction phase. For each distance, the direction of the cylinder was randomly selected from all possible directions that provided a clear path of travel within the boundaries of the physical room. After the participant reached the cylinder's location, it was immediately relocated to the next distance. The interaction phase lasted approximately $3 \mathrm{~min}$, but interaction time was not explicitly measured or controlled. After completion of the interaction phase, the HMD screen turned blank, and the participant was led back to the viewing position before completing the postinteraction walking judgments and size judgments.

Analysis Size judgments were converted to distance judgments using Eq. 1, which assumes size-distance invariance (Gilinsky, 1951; Gogel et al., 1985; Hutchison \& Loomis, 2006; Sedgwick, 1986). When comparing pre- and postinteraction distance judgments, walking judgments and sizebased judgments were expressed as a ratio of the judged to the actual distance and then averaged across actual object distance. This was necessary in order to directly compare the pre- and postinteraction judgments, because object distances varied slightly between the pre- and postinteraction phases of the experiment. A distance judgment ratio of 1.0 indicated veridical performance, and a ratio less than 1.0 indicated distance underestimation.

\section{Results}

Average walking and size-based distance judgments are shown in Fig. 2a and $b$ for illustrative purposes (these data were not directly analyzed as a function of actual distance). The average distance judgment ratios for walking distance judgments and size-based distance judgments, collapsed across actual distances, are shown in Fig. 3. Both the walking and size-based judgments increased as a result of walking through the virtual environment. The preinteraction walking judgment ratios averaged 0.73 , which was significantly less than $1.0, t(12)=7.81, p<.001, d=4.51$. The postinteraction walking judgment ratios averaged 0.82 , which was significantly less than $1.0, t(12)=6.22, p<.001, d=4.17$, but significantly larger than the preinteraction walking judgment ratios, $t(12)=2.91, p=.013, d=1.68$. The preinteraction size-based distance judgment ratios averaged 0.63 , which was significantly less than $1.0, t(12)=4.88, p<.001, d=2.82$. The postinteraction size-based ratios averaged 0.70 , which was 

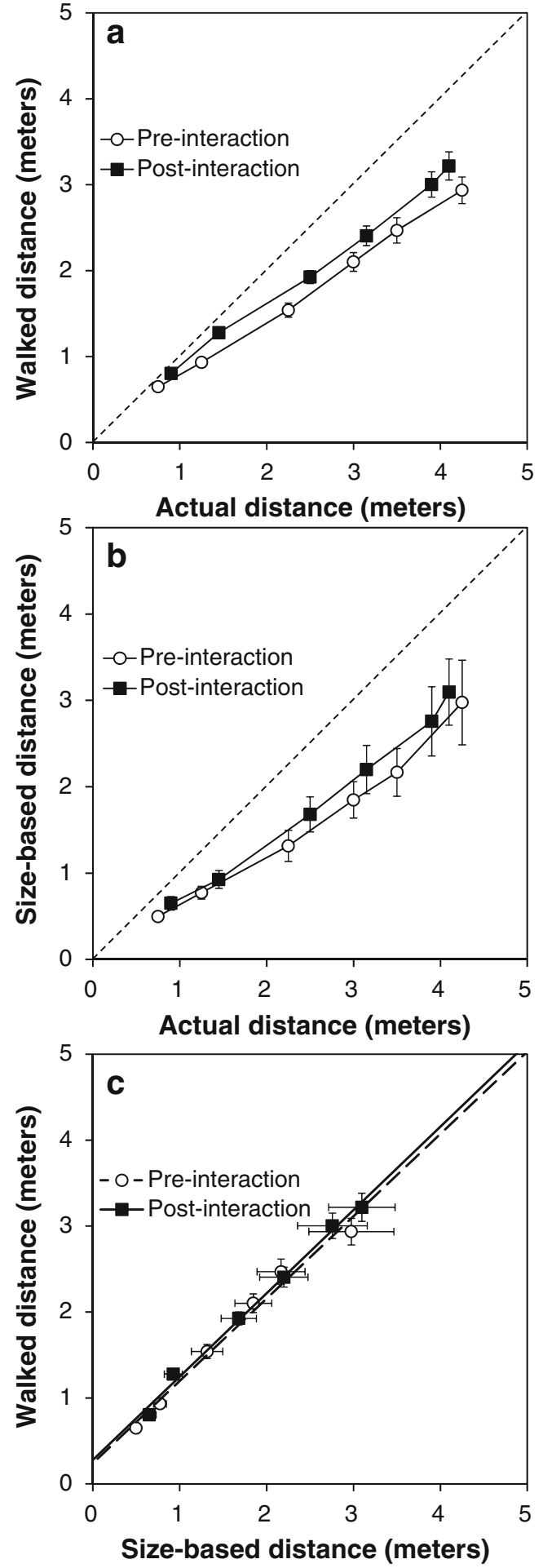

Fig. 2 Mean walked distances (a) and size-based distances (b) as a function of actual distance in Experiment 1. The diagonal lines indicate perfect performance. In panel c, mean walked distances are shown as a function of mean size-based distance in Experiment 1. Separate data points correspond to different actual distances, and the lines show the best-fitting straight lines through the average data. Error bars represent \pm 1 SEM

significantly less than $1.0, t(12)=3.54, p=.004, d=2.04$, but significantly larger than the preinteraction size-based judgment

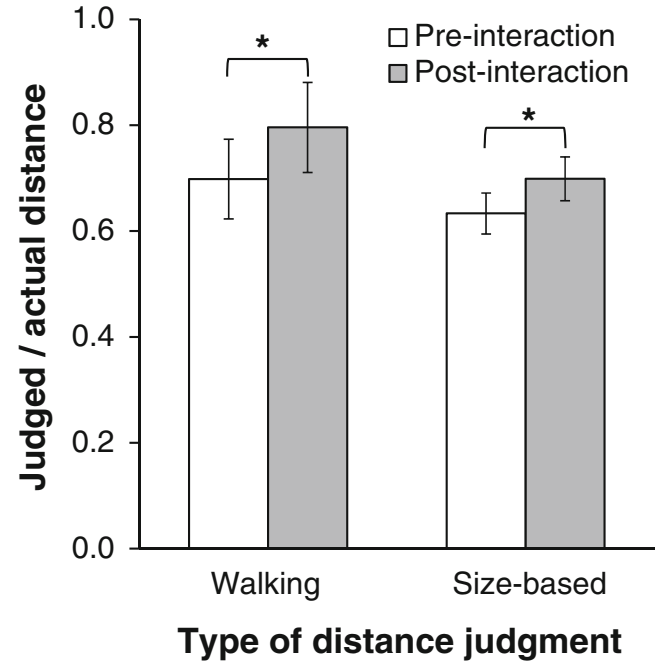

Fig. 3 Mean ratios of judged distance to actual distance in Experiment 1. Participants made walking distance judgments and size judgments (which were later converted to size-based distance judgments) before and after interacting with a virtual environment by walking. Error bars represent $\pm 1 S E M$. The asterisks indicate significant differences between the conditions at $p<.05$

ratios, $t(12)=2.32, p=.039, d=1.34$. The size-based distance judgments $(M=0.67, S E=0.08)$ did not differ significantly from the walking distance judgments $(M=0.78, S E=0.03)$, $t(12)=1.31$. The correlation between the changes (from pre- to postinteraction) in walking distance judgments and size-based distance judgments was positive but did not reach statistical significance, $r(11)=.33$.

Average walked distances are shown as a function of average size-based distance in Fig. 2c, along with the bestfitting lines. Data are plotted separately for the pre- and postinteraction judgments because the actual distances used in pre- and postinteraction phases were slightly different. The relationship between the two response measures can be used to evaluate the degree to which the two measures were affected by object distance and by other independent variables (notably, walking interaction in the present experiment). Under the size-distance invariance hypothesis, perceived size is linearly related to perceived distance (see Eq. 1). The withinparticipant product moment correlations between the walking and size-based judgments averaged $.97(S E=0.004)$ and .98 $(S E=0.003)$ for the pre- and postinteraction judgments, respectively. The between-participant product moment correlations (using the average data points shown in Fig. 2c) were .98 and .99 for the pre- and postinteraction judgments, respectively. The slopes of the best-fitting lines, fit separately for individual participants, averaged $1.31(S E=0.23)$ and 1.18 $(S E=0.11)$ for the pre- and postinteraction judgments, respectively, and these slopes were not significantly different from $1.0, t \mathrm{~s}<1.4, p \mathrm{~s}>.20$. The slopes of the best-fitting lines using the average data shown in Fig. 2c were 0.96 and 0.97 for the pre- and postinteraction judgments, respectively. 


\section{Discussion}

Walking through a virtual environment displayed on an HMD caused an increase in walking distance judgments and also in size-based distance judgments made within the virtual environment. These findings can be accounted for by the rescaling hypothesis, but not by explicit strategies or by the recalibration hypothesis. According to the rescaling hypothesis, walking through the virtual environment changes the perceived scale of the virtual environment. Such a change to the underlying perceptual representation should affect all distance judgments based on that percept, including walking judgments and sizebased judgments, and this prediction is consistent with the results of Experiment 1. According to the recalibration hypothesis, walking through the virtual environment modifies the coupling between visual self-motion and physical/imagined translation, which is specific to responses involving translational movements. Therefore, interaction should only have affected the walking distance judgments and should not have affected size-based distance judgments, which is contrary to the results of Experiment 1.

Of the two formulations of the explicit strategy, the response modification strategy can explain the improvement in postinteraction walking judgments because the walking response could be modified on the basis of feedback during interaction. However, the response modification strategy cannot explain the postinteraction improvement in verbal size judgments, because no feedback was provided regarding that particular response. The representation modification strategy can also explain improved postinteraction walking judgments, because the perceptual representation could be corrected on the basis of feedback during interaction, and all responses would be direction at the corrected location. However, it is unlikely that the corrected representation would also result in an increase in size judgments. Increasing perceived distance causes corresponding increases in perceived size, yet the layperson's expectation (based on informal polling by the first author) is that increasing perceived distance to an object would cause a decrease in the object's perceived size. Therefore, neither explicit strategy seems well-suited to account for the findings of Experiment 1.

Across both judgment types, walking through the virtual environment caused an $11 \%$ increase in distance judgments relative to preinteraction judgments. The magnitude of this effect is small as compared to the approximately $80 \%$ increase reported in previous work using similar methods (Richardson \& Waller, 2005, 2007; Waller \& Richardson, 2008). However, preinteraction walking judgments in Experiment 1 produced distance judgment ratios that were larger than those reported in past studies ( 0.73 versus approximately 0.55$)$, which reduced the potential to observe a large increase in postinteraction judgments. Mohler et al. (2006) reported preinteraction distance judgments that were similar to those found in Experiment 1, and postinteraction judgments in their study were near veridical. Differences in display technology might account for differences in preinteraction judgment ratios, and might also account for differences in the efficacy of interaction with the virtual environment. Furthermore, allowance of head movements during distance judgments in the present study could have provided participants with motion parallax, even during preinteraction judgments, which could have allowed them to calibrate distance and size judgments to some degree (Wexler \& van Boxtel, 2005). Whether previous studies on space perception in virtual reality have allowed such head movements is unclear.

If interaction with the virtual environment caused rescaling of perceived space, then participants who experienced greater rescaling should have evidenced greater changes in both walking and size-based distance judgments. However, changes in the two response measures were not significantly correlated with one another. The rescaling hypothesis is the only one that makes predictions about this correlation, but the lack of correlation is difficult to interpret, and opens the door for alternative proposals including independent mechanisms through which walking interaction affects walking distance judgments and size judgments.

The linear relationship between walking and size-based distance judgments indicates that both judgment types were influenced by the same underlying variable: perceived distance. Despite the increase in size-based and walking distance judgments after interaction, the linear functions relating sizebased and walking distance judgments are remarkably similar before and after interaction. This finding supports the size-distance invariance hypothesis, and bears close resemblance to the tight correspondence between walking judgments and verbal judgments of perceived distance reported by Philbeck and Loomis (1997).

Research on the effects of interaction with a virtual environment indicates that body-based movement through the environment with accompanying feedback is both necessary (Waller \& Richardson, 2008) and sufficient (Mohler et al., 2006) to cause an increase in postinteraction distance judgments. However, it is unclear whether any body-based interaction, such as reaching or throwing, can cause an increase in postinteraction distance judgments, or whether walking is necessary. Therefore, Experiment 2 examined the limits of body-based interactions by evaluating whether interaction with a virtual environment through reaching would cause an increase in walking and/or size-based distance judgments.

\section{Experiment 2}

Participants in Experiment 2 made direct blind-walking judgments and size judgments before and after interacting with a virtual environment by reaching. The reaching task required 
the participant to move his or her hand in order to manipulate cylinders in the virtual environment. Continuous visual feedback indicated the location of the hand during reaching. If any body-based interaction can cause an increase the scale of perceived space, then walking and size-based distance judgments should increase after the reaching interaction. However, if rescaling of perceived space relies on feedback from walking interaction, then distance judgments should be unaffected by reaching interaction.

\section{Method}

Participants A group of 17 students at Iowa State University participated in exchange for course credit. Two participants withdrew from the experiment after experiencing simulator sickness. The data from one participant were removed from all analyses due to size judgments that were not highly correlated with actual object size $(r=.40)$ relative to the average correlation across all other participants $(M=.96$, $S E=.01)$.

Stimuli, design, and procedure The stimuli, design, and procedure were identical to those of Experiment 1, with the exception of the interaction phase. Prior to interaction, each participant was guided without vision to the center of the room. There, the participant was instructed to place his or her feet on either side of a small wooden block affixed to the floor in order to prevent movement away from the room center during the interaction. Once in position, the participant was given a hand-held light that was tracked by the optical tracking system used to track head position. In the virtual environment, a gray cylinder $(0.1-\mathrm{m}$ diameter, $0.3-\mathrm{m}$ height $)$ appeared at the location of the hand-held light and was dynamically repositioned when the light was moved. One red and one blue cylinder also appeared in front of the participant (see Fig. 4). Both cylinders were $0.1 \mathrm{~m}$ in diameter, and the cylinder height was dynamically scaled to match the participant's eye height. The participant's goal during the interaction phase was to drag the red cylinder to the location of the blue cylinder. This was accomplished by moving the hand-held light until the hand contacted the red cylinder, after which the location of the red cylinder was linked to the location of the hand. The participant then moved the hand-held light until the red cylinder contacted the blue cylinder, after which the red and blue cylinders moved to new locations and the process was repeated. At the beginning of each reaching trial, the red and blue cylinders were placed at locations ranging between $\pm 90^{\circ}$ relative to the participant's facing direction and at distances $50 \%-100 \%$ of the participant's maximum arm length. The total time spent on the reaching task was $3 \mathrm{~min}$, which had

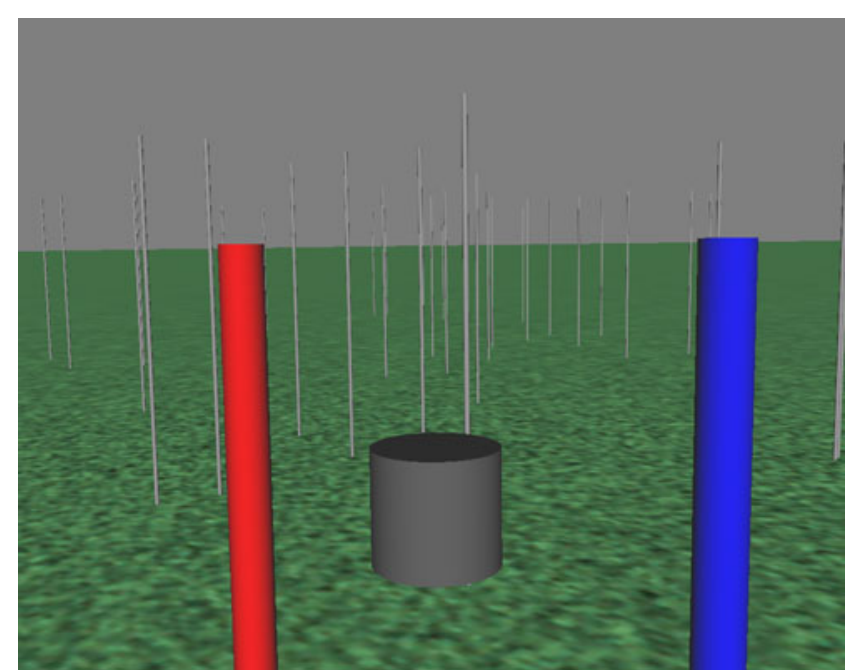

Fig. 4 Perspective view of the virtual environment during the reaching interaction in Experiment 2. The red and blue cylinders represent the reaching targets, and the gray cylinder represents the position of the participant's hand. The radii of the three cylinders were physically equivalent, but the gray cylinder is larger in the image because it is relatively closer to the position from which the screenshot was taken

been the estimated length of the Experiment 1 interaction phase.

\section{Results}

Average walking and size-based distance judgments as a function of actual distance are shown in Fig. 5a and b for illustrative purposes (these data were not directly analyzed as a function of actual distance). The average distance judgment ratios for walking distance judgments and size-based distance judgments, collapsed across actual distances, are shown in Fig. 6. Neither walking nor size-based judgments were affected by the reaching interaction. The preinteraction walking judgment ratios averaged 0.81 , which was significantly less than $1.0, t(13)=5.67, p<.001, d=3.15$. The postinteraction walking judgment ratios averaged 0.81 , again significantly less than $1.0, t(13)=4.70, p<.001, d=2.61$, and also not significantly different from the preinteraction walking judgment ratios. The preinteraction size-based distance judgment ratios averaged 0.59 , which was significantly less than $1.0, t(13)=6.99, p<.001, d=3.88$. The postinteraction sizebased ratios averaged 0.55 , which was also significantly less than $1.0, t(13)=8.17, p<.001, d=4.53$, and not significantly different from the preinteraction size-based judgment ratios. Furthermore, the size-based distance judgments were significantly smaller than the walking distance judgments, $t(13)=$ $4.36, p=.001, d=2.42$.

The preinteraction walking judgments in Experiment 2 were nominally larger than those in Experiment 1, but this difference was not statistically significant, $t(25)=1.61$, 

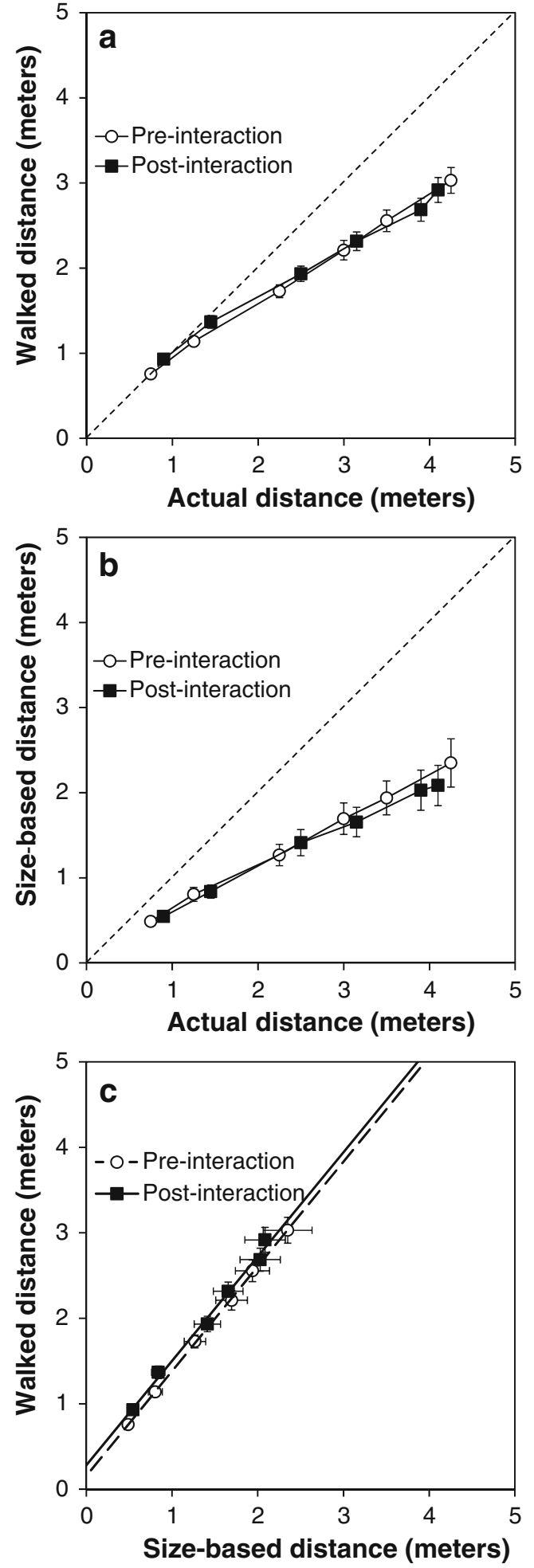

Fig. 5 Mean walked distances (a) and size-based distances (b) as a function of actual distance in Experiment 2. The diagonal lines indicate perfect performance. In panel c, mean walked distances are shown as a function of mean size-based distance in Experiment 2. Separate data points correspond to different actual distances, and the lines show the best-fitting straight lines through the average data. Error bars represent \pm 1 SEM

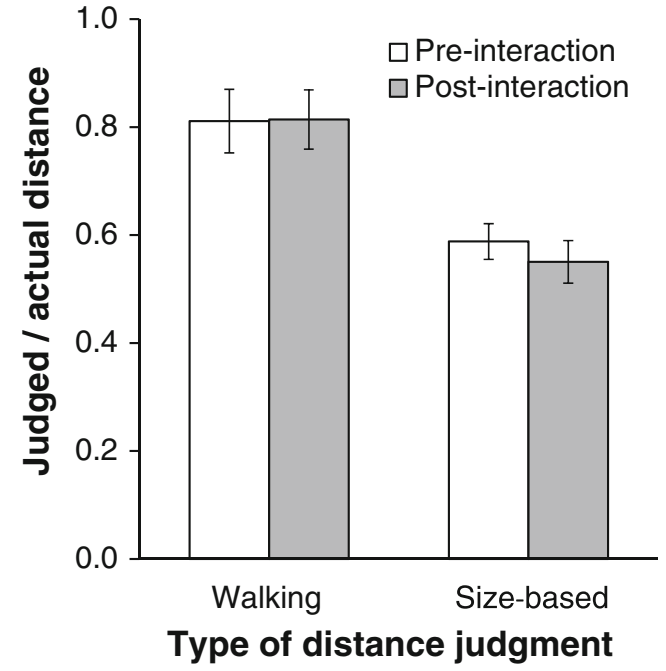

Fig. 6 Mean ratios of judged distance to actual distance in Experiment 2. Participants made walking distance judgments and size judgments (which were later converted to size-based distance judgments) before and after interacting with a virtual environment by reaching. Error bars represent $\pm 1 S E M$

$p=.12$. The preinteraction size judgments in Experiment 2 were nominally smaller than those in Experiment 1, but this difference was also not statistically significant, $t(25)=0.48, p=.64$.

Average walked distances are shown as a function of average size-based distance in Fig. 5c, along with the bestfitting lines. The within-participants product moment correlations averaged $.97(S E=.008)$ and $.96(S E=.018)$ for the pre- and postinteraction judgments, respectively. The between-participants product moment correlations (using the average data points shown in Fig. 2c) were 1.00 for both the pre- and postinteraction judgments. The slopes of the best-fitting lines, fit separately for individual participants, averaged $1.73(S E=0.42)$ and $1.70(S E=0.44)$ for the preand postinteraction judgments, respectively, and these slopes were not significantly different from $1.0, t \mathrm{~s}<1.75, p \mathrm{~s}>.10$. The slopes of the best-fitting lines using the average data shown in Fig. $5 \mathrm{c}$ were 1.22 for both the pre- and postinteraction judgments.

\section{Discussion}

Interacting with a virtual environment by reaching did not influence subsequent distance judgments, regardless of whether those judgments were blind-walking judgments or size-based judgments. These findings serve to identify the boundary conditions for the effects of interaction with a virtual environment on subsequent distance judgments. It seems that not all body-based interactions are sufficient to cause an increase in distance judgments, and that walking may be a necessary condition. Further work will be needed to determine whether the effects of walking through the virtual 
environment also extend to other types of physical selfgenerated movement through the environment, such as riding a bicycle.

In addition to evaluating an alternative mode of physical interaction, in Experiment 2 we also addressed the potential criticism that the difference between the pre- and postinteraction distance judgments reported in Experiment 1 was due to practice. If the effect of interaction in Experiment 1 was actually a practice effect, whereby increased familiarity with judging distances might lead to larger distance judgments, then the same effect should have been observed in Experiment 2. However, we found no evidence of an increase in postinteraction distance judgments in Experiment 2. Furthermore, trial number and judged distance were uncorrelated, indicating that practice with the judgment tasks did not cause an increase in distance judgments. ${ }^{2}$

The tight linear relationship between walking and sizebased distance judgments indicates that both judgment types were influenced by the perceived object distance, providing further support for the size-distance invariance hypothesis.

\section{General discussion}

Past work has indicated that egocentric distances in virtual environments are underperceived by up to $50 \%$ (Loomis \& Knapp, 2003), and that walking through a virtual environment with feedback causes an increase in distance judgments (Mohler et al., 2006; Richardson \& Waller, 2005, 2007; Waller \& Richardson, 2008). The present project was designed to determine the mechanism through which walking in a virtual environment causes an increase in distance judgments within the virtual environment, and to identify the boundary conditions of this effect.

According to the recalibration hypothesis, walking through the virtual environment with visual feedback modifies the coupling between visual input and response output (Rieser, 1999; Rieser et al., 1995). In the context of Experiment 1, the recalibration hypothesis predicts that walking interaction will alter the relationship between perceived walking speed and perceived translational speed through the environment, which should cause an increase in postinteraction blind-walking distance judgments. However, responses that do not involve translation will be unaffected by the walking interaction, and therefore postinteraction verbal size judgments would be no different from preinteraction verbal size judgments.

According to the rescaling hypothesis, walking through the virtual environment changes the scale of perceived space by altering the underlying perceptual representation. During

\footnotetext{
${ }^{2}$ Correlations between trial number and judged distance were calculated separately for the pre- and postinteraction phases and for the walking and size-based distance judgments in both experiments. The average correlation across those factors was .006.
}

interaction, participants receive feedback that the environment is actually larger than it was initially perceived to be, resulting in a global rescaling of the perceptual representation. The exact mechanism of this rescaling is unclear. One possibility is that interaction feedback might directly cause participants to rescale their perceptual representations, similar to the expansion of perceived space that occurs after reaching with an elongated tool (Witt, Proffitt, \& Epstein, 2005). Another possibility is that interaction feedback results in perceptual learning, whereby participants learn to attend to distance cues that had previously been ignored or weighted incorrectly (Waller \& Richardson, 2008). Regardless of the mechanism, the rescaling hypothesis predicts that walking through the virtual environment will cause an increase in the scale of the perceived environment and a corresponding increase in all types of distance judgments (e.g., walking, throwing, and verbal reporting) and also size judgments. In Experiment 1, walking through the virtual environment caused an increase in walking distance judgments and size-based distance judgments, thereby providing support for the rescaling hypothesis.

Neither of the two formulations of the explicit strategy - the response modification strategy and the representation modification strategy - is well-suited to describe the results of Experiment 1 . The response modification strategy fails to account for the generalization of feedback during walking interaction to size judgments performed after the interaction. The representation modification strategy also fails to explain the improved size judgments, since explicit modification of the egocentric distance is unlikely to also involve modification of size (given the layperson's understanding of the relationship between perceived size and perceived distance). Therefore, neither explicit strategy seems well-suited to account for the findings of Experiment 1.

Although their results could not distinguish between the recalibration and rescaling hypotheses, Waller and Richardson (2008) preferred the recalibration hypothesis over the rescaling hypothesis because physical walking was found to be necessary in order to elicit an increase in postinteraction distance judgments, and simulated visual walking without concomitant physical walking was insufficient. However, the importance of physical movement may be due to its role in providing feedback about walked distance, rather than its role in perceptual-motor recalibration. In other words, the errorcorrective signal when walking to a location with vision relies on visual input indicating that the target has not yet been reached and on body-based input indicating traveled distance. Therefore, the necessity of physical walking may reflect its role in the feedback generated during interaction, but does not directly support the perceptual-motor recalibration hypothesis.

Previous research on the effects of interaction with a virtual environment has been limited to interaction by walking. In Experiment 2, we examined whether walking is necessary, or whether another form of body-based 
interaction-reaching-would elicit the same effect. It was found that interacting with a virtual environment by reaching did not influence subsequent distance judgments, indicating that not all body-based interactions are sufficient to cause an increase in distance judgments, and that walking (or possibly other translational actions, such as crawling; Withagen \& Michaels, 2002) may be a necessary condition. However, at least two alternative explanations are based on the distances used during interaction and testing. First, a rescaling of perceived space caused by interaction may be specific to the range of distances engaged during interaction. In the interaction phase of Experiment 1, participants walked egocentric distances that were similar to the distances tested during the postinteraction judgments. In contrast, in the interaction phase of Experiment 2, participants reached to locations placed within arm's reach, and the distances tested during postinteraction judgments were much larger. Therefore, it is possible that rescaling occurred within reaching distance, but that the stimuli used for distance judgments covered a different range of egocentric distances that had not been rescaled. Second, rescaling of perceived space may depend on the distinction between peripersonal space (within reaching distance) and extrapersonal space (beyond reaching distance), which have distinct neural representations (Delevoye-Turrell, Bartolo, \& Coello, 2010; Halligan \& Marshall, 1991; Previc, 1998; Vuilleumier, Valenza, Mayer, Reverdin \& Landis, 1998). Further work will be needed to evaluate whether rescaling of perceived space generalizes to distances outside the range encountered during interaction and also across the boundaries of peripersonal and extrapersonal space.

Richardson and Waller (2005) reported that judgments of egocentric and exocentric distance in a virtual environment were selectively affected by egocentric and exocentric feedback. Although the reaching interaction in Experiment 2 was primarily intended to provide feedback about egocentric distance, it is possible that the exocentric component of the reaching task was one reason that the reaching interaction failed to influence postinteraction egocentric distance judgments. Both stages of the reaching interaction task (reaching for the red post and then dragging it to the location of the blue post) required movement of the hand toward a target object. Such reaching movements with visual feedback are thought to involve both egocentric and exocentric components (Bingham \& Pagano, 1998). The presence of exocentric reaching feedback may have reduced the effectiveness of the egocentric reaching feedback, but past work in the domain of distance perception has typically isolated these sources, making extrapolation to the present results speculative.

The walking interaction used in Experiment 1 involved continuous walking to a sequence of targets. In contrast, the interaction used in previous studies on this topic (e.g., Waller \& Richardson, 2008) involved returning to a single start location prior to each interaction. It is possible that the continuous walking interaction provided participants with information about the scale of the walking space in the virtual environment relative to the physical lab space that they had experienced prior to entering the virtual environment. However, participants were never provided with explicit information linking the virtual and physical spaces, and the space in which participants walked during the interaction was smaller than the physical lab confines (so as to reduce the risk of participants running into physical objects). Therefore, attempts to relate the virtual space to the physical space on the basis of interaction would likely create a very inaccurate representation of the virtual space.

Size-based distance judgments were smaller than walking distance judgments in Experiment 2, but not in Experiment 1 , and the cause is unclear. Although the gray sphere used for size judgments was distinct from the cylinder used for walking distance judgments, neither object possessed additional monocular distance cues. For example, both stimuli lacked shadows, so underperception of egocentric object distance could have resulted in the perception of the object floating above the ground plane (e.g., Wu, Ooi, \& He, 2004). The fact that the cylinder subtended a larger vertical visual angle means that it provided a larger binocular disparity than did the sphere, because the disparity between the top of the cylinder and the background was quite large relative to the disparity between the bottom of the cylinder or the sphere (which did not extend vertically to the horizon) and the background. However, this effect was probably small, since the difference between the judgment types only appeared in Experiment 2.

The close correspondence between walking and sizebased distance judgments across both experiments supports the size-distance invariance hypothesis (Gilinsky, 1951; Gogel et al., 1985; Hutchison \& Loomis, 2006; Sedgwick, 1986), whereby perceived size is linearly related to perceived distance and the perceived angular size of the target object. The evidence from Experiment 1 is particularly compelling, since the linear relationship characterizes both pre- and postinteraction judgments, despite the change in perceived distance caused by interaction. Although an evaluation of size-distance invariance was not a primary goal of the present project, these data contribute to the debate about this invariance and indicate that size-based distance is a valid measure for studying distance perception in virtual reality.

The ability to rescale perceived space in virtual environments by walking through the environment presents an intriguing tool for virtual reality simulations requiring accurate space perception. However, postinteraction walking judgments in Experiment 1 were only $82 \%$ of the modeled distance, leaving considerable room for improvement. Additional time spent interacting might eventually result in distance judgments that approached $100 \%$ of the modeled 
distance, as has been indicated in other, similar studies (e.g., Waller \& Richardson, 2008). Given that walking interaction is not always feasible, and that interaction does not always result in veridical distance judgments, it is clear that further research on the effects of interaction should occur in parallel with research to identify the deficient cues to distance in virtual environments.

\section{References}

Antis, S. (1995). Aftereffects from jogging. Experimental Brain Research, 103, 476-478.

Bingham, G. P., \& Pagano, C. C. (1998). The necessity of a perceptionaction approach to definite distance perception: Monocular distance perception to guide reaching. Journal of Experimental Psychology. Human Perception and Performance, 24, 145-168.

Bodenheimer, B., Meng, J., Wu, H., Narasimham, G., Rump, B., McNamara, T. P., . . Rieser, J. J. (2007). Distance estimation in virtual and real environments using bisection. In C. Wallraven \& V. Sundstedt (Eds.), Proceedings of the 4th Symposium on Applied Perception in Graphics and Visualization (pp. 35-40). New York, NY: ACM.

Bruggeman, H., Pick, H. L., Jr., \& Rieser, J. J. (2005). Learning to throw on a rotating carousel: Recalibration based on limb dynamics and projectile kinematics. Experimental Brain Research, 163, 188-197.

da Silva, J. A. (1985). Scales for perceived egocentric distance in a large open field: Comparison of thee psychophysical methods. The American Journal of Psychology, 98, 119-144.

Delevoye-Turrell, Y., Bartolo, A., \& Coello, Y. (2010). Motor representation and the perception of space: Perceptual judgments of the boundary of action space. In N. Gangopadhyay (Ed.), Perception, action and consciousness (pp. 217-242). Oxford, UK: Oxford University Press.

Durgin, F. H., Pelah, A., Fox, L. F., Lewis, J., Kane, R., \& Walley, K. A. (2005). Self-motion perception during locomotor recalibration: More than meets the eye. Journal of Experimental Psychology. Human Perception and Performance, 31, 398-419.

Eby, D. W., \& Loomis, J. M. (1987). A study of visually directed throwing in the presence of multiple distance cues. Perception \& Psychophysics, 41, 308-312.

Epstein, W., \& Landauer, A. A. (1969). Size and distance judgments under reduced conditions of viewing. Perception \& Psychophysics, 6, 269-272.

Epstein, W., Park, J., \& Casey, A. (1961). The current status of the sizedistance hypotheses. Psychological Bulletin, 58, 491-514.

Foley, J. M. (1977). Effect of distance information and range on two indices of visually perceived distance. Perception, 6, 449-460.

Gibson, E. J., \& Bergman, R. (1954). The effect of training on absolute estimation of distance over the ground. Journal of Experimental Psychology, 48, 473-482.

Gilinsky, A. S. (1951). Perceived size and distance in visual space. Psychological Review, 58, 460-482.

Gogel, W. C. (1969). The sensing of retinal size. Vision Research, 9, 1079-1094.

Gogel, W. C. (1971). The validity of the size-distance invariance hypothesis with cue reduction. Perception \& Psychophysics, 9, 92-94.

Gogel, W. C., Loomis, J. M., Newman, N. J., \& Sharkey, T. J. (1985). Agreement between indirect measures of perceived distance. Perception \& Psychophysics, 37, 17-27.
Gogel, W. C., \& Tietz, J. D. (1973). Absolute motion parallax and the specific distance tendency. Perception \& Psychophysics, 13, 284 292.

Gooch, A. A., \& Willemsen, P. (2002). Evaluating space perception in NPR immersive environments. In Proceedings of the 2nd International Symposium on Non-Photorealistic Animation and Rendering (pp. 105-110). New York, NY: ACM.

Halligan, P. W., \& Marshall, J. C. (1991). Left neglect for near but not far space in man. Nature, 350, 498-500.

Hutchison, J. J., \& Loomis, J. M. (2006). Does energy expenditure affect the perception of egocentric distance? A failure to replicate Experiment 1 of Proffitt, Stefanucci, Banton, and Epstein (2003). Spanish Journal of Psychology, 9, 332-339.

Kelly, J. W., Beall, A. C., \& Loomis, J. M. (2004). Perception of shared visual space: Establishing common ground in real and virtual environments. Presence: Teleoperators and Virtual Environments, $13,442-450$.

Knapp, J. M., \& Loomis, J. M. (2004). Limited field of view of headmounted displays is not the cause of distance underestimation in virtual environments. Presence: Teleoperators and Virtual Environments, $13,572-577$.

Kuhl, S. A., Thompson, W. B., \& Creem-Regehr, S. H. (2009). HMD calibration and its effects on distance judgments. ACM Transactions on Applied Perception, 6, 19:1-19:20.

Loomis, J. M., da Silva, J. A., Fujita, N., \& Fukusima, S. S. (1992). Visual space perception and visually directed action. Journal of Experimental Psychology. Human Perception and Performance, 18, 906-921. doi:10.1037/0096-1523.18.4.906

Loomis, J. M., Klatzky, R. L., \& Giudice, N. A. (2013). Representing 3-D space in working memory: Spatial images from vision, hearing, touch, and language. In S. Lacey \& R. Lawson (Eds.), Multisensory imagery: Theory and applications (pp. 131-155). New York, NY: Springer.

Loomis, J. M., Klatzky, R. L., Philbeck, J. W., \& Golledge, R. G. (1998). Assessing auditory distance perception using perceptually directed action. Perception \& Psychophysics, 60, 966-980.

Loomis, J. M., \& Knapp, J. M. (2003). Visual perception of egocentric distance in real and virtual environments. In L. J. Hettinger \& M. W. Haas (Eds.), Virtual and adaptive environments (pp. 21-46). Mahwah NJ: Erlbaum.

Loomis, J. M., \& Philbeck, J. W. (2008). Measuring perception with spatial updating and action. In R. L. Klatzky, M. Behrmann, \& B. MacWhinney (Eds.), Embodiment, ego-space, and action (pp. 143). Mahwah, NJ: Erlbaum.

Messing, R., \& Durgin, F. H. (2005). Distance perception and the visual horizon in head-mounted displays. ACM Transactions on Applied Perception, 2, 234-250.

Mohler, B. J., Creem-Regehr, S. H., \& Thompson, W. B. (2006). The influence of feedback on egocentric distance judgments in real and virtual environments. In ACM SIGGRAPH Symposium on Applied Perception in Graphics and Visualization (pp. 9-14). Washington, DC: ACM.

Mohler, B. J., Thompson, W. B., Creem-Regeher, S. H., Willemsen, P., Pick, H. L., \& Rieser, J. J. (2007). Calibration of locomotion due to visual motion in a treadmill-based virtual environment. $A C M$ Transactions on Applied Perception, 4, 20-32.

Ooi, T. L., Wu, B., \& He, Z. J. (2001). Distance determined by the angular declination below the horizon. Nature, 414, 197-200.

Oyama, T. (1974). Perceived size and perceived distance in stereoscopic vision and an analysis of their causal relations. Perception \& Psychophysics, 16, 175-181.

Philbeck, J. W., \& Loomis, J. M. (1997). Comparison of two indicators of visually perceived egocentric distance under full-cue and reduced-cue conditions. Journal of Experimental Psychology. Human Perception and Performance, 23, 72-85. doi:10.1037/00961523.23.1.72 
Philbeck, J. W., Loomis, J. M., \& Beall, A. C. (1997). Visually perceived location is an invariant in the control of action. Perception \& Psychophysics, 59, 601-612.

Previc, F. H. (1998). The neuropsychology of 3-D space. Psychological Bulletin, 124, 123-164.

Richardson, A. R., \& Waller, D. (2005). The effect of feedback training on distance estimation in virtual environments. Applied Cognitive Psychology, 19, 1089-1108.

Richardson, A. R., \& Waller, D. (2007). Interaction with an immersive virtual environment corrects users' distance estimates. Human Factors, 49, 507-517.

Rieser, J. J. (1999). Dynamic spatial orientation and the coupling of representation and action. In R. G. Golledge (Ed.), Wayfinding behavior: Cognitive mapping and other spatial processes (pp. 168-190). Baltimore, MD: Johns Hopkins University Press.

Rieser, J. J., Ashmead, D. H., Talor, C. R., \& Youngquist, G. A. (1990). Visual perception and the guidance of locomotion without vision to previously seen targets. Perception, 19, 675-689.

Rieser, J. J., Pick, H. L., Ashmead, D. H., \& Garing, A. E. (1995). Calibration of human locomotion and models of perceptual-motor organization. Journal of Experimental Psychology. Human Perception and Performance, 21, 480-497.

Sedgwick, H. A. (1986). Space perception. In K. R. Boff, L. Kaufman, \& J. P. Thomas (Eds.), Handbook of perception and human performance: Vol I. Sensory processes and perception (pp. 21.121.57). New York, NY: Wiley.

Sinai, M. J., Ooi, T. L., \& He, Z. J. (1998). Terrain influences the accurate judgement of distance. Nature, 395, 497-500.

Steinicke, F., Bruder, G., Ries, B., Hinrichs, K. H., Lappe, M., \& Interrante, V. (2009). Transitional environments enhance distance perception in immersive virtual reality systems. In Proceedings of the 6th Symposium on Applied Perception in Graphics and Visualization (pp. 19-26). New York, NY: ACM.

Thompson, W. B., Willemsen, P., Gooch, A. A., Creem-Regehr, S. H., Loomis, J. M., \& Beall, A. C. (2004). Does the quality of the computer graphics matter when judging distances in visually immersive environments. Presence: Teleoperators and Virtual Environments, 13, 560-571.
Tresilian, J. R., Mon-Williams, M., \& Kelly, B. M. (1999). Increasing confidence in vergence as a cue to distance. Proceeding of the Royal Society B, 266, 39-44.

Vuilleumier, P., Valenza, N., Mayer, E., Reverdin, A., \& Landis, T. (1998). Near and far visual space in unilateral neglect. Annals of Neurology, 43, 406-410.

Waller, D., \& Richardson, A. R. (2008). Correcting distance estimates by interacting with immersive virtual environments: Effects of task and available sensory information. Journal of Experimental Psychology. Applied, 14, 61-72.

Wexler, M., \& van Boxtel, J. J. A. (2005). Depth perception by the active observer. Trends in Cognitive Sciences, 9, 431438.

Willemsen, P., Colton, M. B., Creem-Regehr, S. H., \& Thompson, W. B. (2009). The effects of head-mounted display mechanical properties and field-of-view on distance judgments in virtual environments. ACM Transactions on Applied Perception, 6, 8:1-8:14.

Willemsen, P., Gooch, A. A., Thompson, W. B., \& Creem-Regehr, S. H. (2008). Effects of stereo viewing conditions on distance perception in virtual environments. Presence: Teleoperators and Virtual Environments, 17, 91-101.

Withagen, R., \& Michaels, C. F. (2002). The calibration of walking transfers to crawling: Are action systems calibrated? Ecological Psychology, 14, 223-234.

Witmer, B. G., \& Sadowski, W. J. (1998). Nonvisually guided locomotion to a previously viewed target in real and virtual environments. Human Factors, 50, 478-488.

Witt, J. K., Proffitt, D. R., \& Epstein, W. (2005). Tool use affects perceived distance, but only when you intend to use it. Journal of Experimental Psychology. Human Perception and Performance, 31, 880-888. doi:10.1037/0096-1523.31.5.880

Wu, B., Ooi, T. L., \& He, Z. J. (2004). Perceiving distance accurately by a directional process of integrating ground information. Nature, 428 , 73-77.

Ziemer, C. J., Plumert, J. M., Cremer, J. F., \& Kearney, J. K. (2009). Estimating distance in real and virtual environments: Does order make a difference? Attention, Perception, \& Psychophysics, 71, 1095-1106. doi:10.3758/APP.71.5.1096 